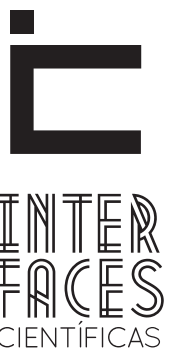

EDUCAÇÃO

\title{
REALIDADE VIRTUAL: CONCEITOS, EVOLUÇÃO, DISPOSITIVOS E APLICAÇ̃̃ES
}

Gessica Palhares Rodrigues ${ }^{1}$

\section{RESUMO}

Este artigo objetiva expor os conceitos sobre Realidade Virtual, visando estabelecer uma interlocução entre alguns conceitos utilizados. Além de trazer uma descrição do seu surgimento e evolução pretende-se ainda, esclarecer termos pertinentes de uma maneira didática e introduzir noções básicas, como o conceito de interação, imersão e envolvimento. 0 texto descreve, também, dispositivos de visualização e interação que são de fundamental importância quando se trata de Realidade Virtual, pois permite ao usuário "entrar" no mundo virtual, sem eles seria impossível a ocorrência dessa interação. Será apresentada também uma visão geral da aplicação de Realidade Virtual em
Cristiane de Magalhães Porto²

diversas áreas, como medicina, arquitetura e militar. 0 presente trabalho pode ser classificado como descritivo e de revisão visto que, propicia informações sobre os assuntos abordados, além da discussão de informações que já haviam sido publicadas. A coleta de material para a construção deste artigo deve-se a pesquisas e estudos feitos além da observação assistemática e participante.

\section{PALAVRAS-CHAVE}

Realidade Virtual. Dispositivos de Visualização. Interação. Aplicação de Realidade Virtual. 


\section{ABSTRACT}

This article aims to present the concepts of Virtual Reality, to establish a dialogue between some concepts used. Besides bringing a description of its emergence and evolution it is also intended clarify relevant terms in a didactical way and introduce some basics as the concept of interaction, immersion and involvement. The text also describes display devices and interaction that are of fundamental importance when it comes Virtual Reality, it allows the user get in the virtual world, without them would be impossible the occurrence of this interaction. Will be also presented an overview of the application of Virtual Reality in various areas such as medicine, architecture and military. This work can be classified as descriptive and review because provides information about the matters discussed beyond the analysis and discussion of information that had already been published. The collect of material for the construction of this article due to research and studies besides the unsystematic and participant observation.

\section{KEYWORDS}

Virtual Reality. Display Devices. Interaction. Application of Virtual Reality.

\section{INTRODUÇ̃̃O}

Apesar de a Realidade Virtual existir a mais de duas décadas ela tem se manifestado significativamente nos últimos anos. Tal aspecto está diretamente conectado com a utilização de equipamentos sofisticados, o que custou um alto preço na sua implementação por um longo período. Na contemporaneidade, com o notável avanço tecnológico e a propagação da indústria de computadores, a Realidade Virtual passou a ser viável e empresas de produtos eletrônicos

\section{RESUMEN}

Este artículo trata de explicar los conceptos de Realidad Virtual, para establecer un diálogo entre algunos de los conceptos utilizados. Además de traer una descripción de su aparición y evolución pretende aclarar los términos relevantes de una manera didáctica e introducir conceptos básicos, como el concepto de interacción, la inmersión y la participación. El texto también describe dispositivos de visualización e interacción que son de importancia fundamental cuando se trata de la realidad virtual, ya que permite al usuario “, entre" el mundo virtual, que sería imposible sin la presencia de esta interacción. También se presentará una visión general de la aplicación de la realidad virtual en diversos campos como la medicina, la arquitectura y militar. Este trabajo puede ser clasificada como descriptiva y revisión visto que proporciona información sobre los temas tratados, así como el análisis y discusión de la información que ya se había publicado. La recolección de material para la construcción de este artículo es la investigación y los estudios, además de la observación sistemática y el participante.

\section{PALABRAS CLAVE}

Realidad Virtual. Dispositivos Ver. Interacción. Aplicación de la Realidad Virtual.

passaram a desenvolver mercadorias para serem utilizadas por ela.

Este trabalho busca abordar o surgimento da Realidade Virtual, conceituando o termo com base em alguns pesquisadores e autores da área. Dispositivos de visualização e interação, como: vídeo-capacetes, sistemas de projeção, luvas de dados e sensores biológicos também serão discutidos aqui. Este documento 
está dividido em temas e subtemas onde estes vão esquadrinhar as informações pertinentes de forma mais objetiva possível.

Para realização deste artigo utilizou-se a pesquisa em laboratório, visando o aprofundamento para a obtenção de uma boa coleta de dados, a fim de selecionar as melhores opções para a criação deste material, além da observação direta participante proporcionada pela interação com ambientes onde existe a RV e o contato com pessoas que a utilizam.

Dessa forma, serão apresentadas três aplicações da Realidade Virtual, mostrando a utilização desta em diversas áreas da ciência, no caso deste artigo os ramos de medicina, arquitetura e aeronáutica.

\section{QUE É REALIDADE VIRTUAL}

Existe uma extensa quantidade de definições sobre realidade virtual, de forma geral, elas fazem referência a uma imersiva e interativa experiência que se baseia em imagens gráficas 3D geradas por computador em tempo real, em outras palavras, é uma simulação de um mundo real, ou apenas imaginário gerada por computador.

Alguns indivíduos não entendem como uma realidade pode ser virtual, por achar que uma coisa virtual é algo que não existe, sendo que Lévy (1996) explica

\subsection{CONCEITOS}

O termo Realidade Virtual (RV) foi inventado no final da década de 1980 por Jaron Lanier, cientista da computação e artista que conseguiu afluir dois conceitos antagônicos em um novo conceito diferenciando assim as simulações tradicionais feitas por computador de simulações envolvendo múltiplos usuários em um ambiente compartilhado (ARAÚJO, 1996). Pimentel (1995) afirma que a Realidade Virtual (RV) é o uso de alta tecnologia para convencer o usuário de que ele se encontra em outra realidade, provocando o seu envolvimento por completo.

Outra definição é o uso de computadores e interfaces com o usuário para criar o efeito de mundos tridi- que virtual não se opõe ao real, mas ao atual. Ele vai ainda mais longe ao afirmar que:

\begin{abstract}
O virtual é como o complexo problemático, o nó de tendências ou de forças que acompanha uma situação, um acontecimento, um objeto ou uma entidade qualquer, e que chama um processo de resolução: a atualização. (LÉVY, 1996, p. 16).
\end{abstract}

Constata-se que o mais importante é entender que o conceito de virtual neste contexto faz referência a uma noção vital do real, da virtude, da potência.

mensionais que incluem objetos interativos com uma forte sensação de presença tridimensional (BRYSON, 1996). Segundo Lévy a interface:

Designa um dispositivo que garante a comunicação
entre dois sistemas informáticos distintos ou um sis-
tema informático e uma rede de comunicação. Nesta
acepção do termo, a interface efetua essencialmente
operações de transcodificação e de administração dos
fluxos de informação. (LÉVY, 1993, p. 176).

Constata-se que a interface utiliza "alavancas" para impulsionar as informações ofertadas, possibilitando uma modalidade nova de leitura e escrita. A internet pode ser vista como uma ilustração, uma 
vez que se encontra equipada com interfaces gráficas atraentes e bem estruturadas, disponibilizando assim o acesso as informações de forma mais didática. Com isso, a interface passa a ser a parte mais significativa da metamorfose ocorrente na sociedade contemporânea, visto que a telepresença encontra-se atualmente como uma marca bastante relevante que a convivência. (PORTO, 2011).

\subsection{Origem e Evolução da RV}

A Realidade Virtual teve seu início na indústria de simulação, com os simuladores de voo que a Força Aérea dos Estados Unidos passou a construir depois da Segunda Guerra Mundial (JACOBSON, 1994).

A indústria de entretenimento também teve a sua importância no surgimento da Realidade Virtual através do simulador conhecido por Sensorama (Figura 1).

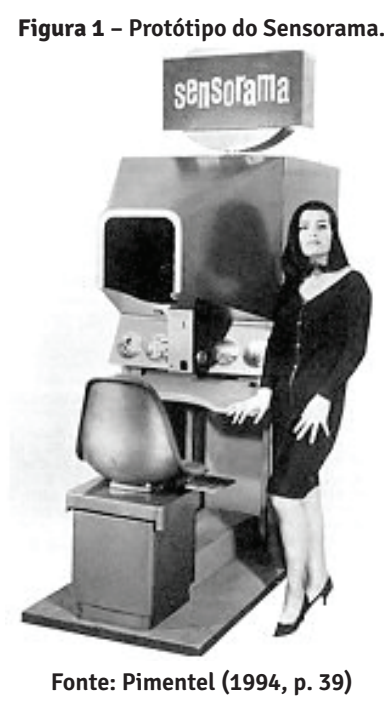

O Sensorama era uma espécie de cabine que permitia ao usuário expor-se a uma combinação de som estéreo, visão tridimensional, vibrações mecânicas, ar movimentado por ventiladores e até aromas, tudo isso
A RV é um "espelho" da realidade física, na qual o indivíduo existe em três dimensões, tem a sensação do tempo real e a capacidade de interagir com o mundo ao seu redor. Os equipamentos de RV simulam essas condições, chegando ao ponto em que o usuário pode "tocar" os objetos de um mundo virtual e fazer com que eles respondam, ou mudem, de acordo com suas ações (VON SCHWEBER, 1995).

proporcionando ao utilizador a participação em uma viagem multissensorial. Embora não tenha sido um sucesso comercial, o invento patenteado por Morton Heilig em 1962 já utilizava um dispositivo para visão estereoscópica, além de ter sido o percursor da imersão do usuário em um ambiente artificial.

Por volta de 1965, Ivan Sutherland apresentou a comunidade cientifica, a ideia de usar computadores para desenhar projetos diretamente em sua tela, utilizando uma caneta ótica, surgindo com isso à computação gráfica. Alguns anos depois, Sutherland tornou-se o percursor do que agora é a indústria de CAD, por ter desenvolvido o primeiro vídeo-capacete totalmente funcional para a computação gráfica no projeto conhecido por "The Ultimate Display”, possibilitando ao usuário enxergar, movimentando a cabeça, diferentes lados de uma construção de arame na forma de um cubo flutuando no espaço. (PIMENTEL, 1995).

Em 1975 Myron Krueger criou o videoplace, onde uma câmera de vídeo capturava a imagem de participantes e projetava-a em 2D numa grande tela, essa técnica ficou conhecida como Realidade Virtual de Projeção. Thomas Furness, em 1982, apresentava para a Força Aérea Americana o Visually Coupled Airbone Systems Simulator (VCASS), conhecido por "Super Cockpit". O VCASS era um simulador que usava vídeo-capacetes e computadores interligados para representar o espaço 3D da cabine de um avião. 0 VCASS era bastante rápido na atualização de imagens 
complexas e possuía alta qualidade na resolução de imagens, porém o custo era um problema: milhões de dólares eram indispensáveis apenas para o capacete.

Aplicando uma nova tecnologia de visores de cristal liquido (LCD) Michael McGreevy, em 1984 na NASA, começou a trabalhar no projeto VIVED (Virtual Environment Display) no qual as imagens seriam estereoscópicas. Em comparação ao VCASS a resolução das imagens era limitada, porém o custo era deveras atrativo. A parte de vídeo e áudio foi montada sobre uma mascara de mergulho usando dois visores de cristal liquido com pequenos alto-falantes acoplados. Em 1985, com o objetivo de incluir nele: luvas de dados, reconhecimento de voz, síntese de som 3D, e dispositivos de feedback tátil; Scott Fisher juntou-se a esse projeto.

\subsection{Imersão, Interação e Envolvimento}

A Realidade Virtual pode ser caracterizada ainda pela integração de três ideias básicas: imersão, interação e envolvimento.

A concepção de imersão está relacionada com o objetivo de mostrar que o usuário, quando imerso no ambiente virtual, pode propiciar-se a sensação de estar dentro do ambiente. Todavia, a identificação da proporção de imersão, é captada pelos dispositivos que transmitem ao utilizador a sensação de entrada no ambiente virtualizado, levando seus sentidos sensoriais e atenção para o que está acontecendo dentro desse espaço, com isso isola-o do mundo exterior permitindo-lhe manipular e explorar naturalmente os objetos ao invés de ser apenas um observador.

A interação está associada à capacidade de o computador detectar as entradas do usuário e modificar em tempo real o mundo virtual e as ações sobre ele. As pessoas gostam de uma boa simulação e de ver as cenas/situações mudarem de acordo aos seus comandos, este é um dos motivos das pessoas utilizarem a RV para o divertimento, visto que a interação
Em 1986 a NASA já possuía um ambiente virtual que permitia aos usuários escutar fala sintetizada e som 3D, ordenar comandos pela voz além de manipular objetos virtuais por meio do movimento das mãos, utilizando uma luva especial chama de "DataGlove", desenvolvida com sensores de fibra ótica.

A percepção de que os projetos da NASA poderiam se tornar equipamentos comercializáveis deu inicio a inúmeros programas de pesquisa em Realidade Virtual em todo o globo. De empresas de software à grandes corporações de informática começaram a desenvolver e vender serviços e produtos voltados para RV.

A AutoDesk, em 1989, apresentou o primeiro sistema de Realidade Virtual voltado para computadores pessoais.

é um dos princípios básicos dos videogames. Para parecer ainda mais realista, o ambiente virtual inclui objetos simulados e existe também a inserção de sons ambientais e sons associados a objetos específicos.

Para ilustrar este tópico posso citar um projeto desenvolvido por Ralph Kistler e Jan M. Sieber, o Monkey Business, que consiste em uma instalação interativa, onde um macaco de pelúcia pendurado na parede imita os movimentos e gestos do usuário. Os movimentos realizados em frente ao macaco são capturados por uma câmera Kinect, um computador oculto analise as informações obtidas e converte-las em movimento enviando logo em seguida para um chip situado no interior do corpo do boneco possibilitando assim a movimentação do corpo do macaco. (PLAYNGIVE, 2011).

O Envolvimento, por sua vez, está ligado ao grau de estimulação para o comprometimento de uma pessoa com determinada atividade, podendo ser ativo (participar de um jogo, visualizar um ambiente virtual) ou passivo (ler um livro, participar de uma cirurgia virtual). 


\section{DISPOSITIVOS DE SAÍDA E ENTRADA}

$\mathrm{Na}$ tentativa de garantir que o usuário se sinta imerso e possa interagir com o ambiente virtual, as tecnologias de saída e entrada de dados associada

\subsection{DISPOSITIVOS DE SAÍDA DE DADOS}

A grande massa das aplicações de RV baseia-se no isolamento dos sentidos, principalmente a visão. $\mathrm{Na}$ maioria das vezes, adentrar um mundo virtual exige a utilização de um equipamento, para isolar o usuário

\subsubsection{DISPOSITIVOS VISUAIS}

Os dispositivos visuais e a qualidade das imagens geradas por eles são uma forte influencia a percepção do nível de imersão de um sistema de RV.

Existem duas categorias de dispositivos visuais, a primeira delas é composta pelos vídeo-capacetes (HMDs) e head-coupled displays (dispositivos que usam braços mecânicos para permanecer posicio-

\section{- VÍDEO-CAPACETES [HMDS]}

0 vídeo-capacete (Head-Mouted Display, HMD) é um dos dispositivos de interface para RV mais populares por se tratar do dispositivo de saída que mais isola o usuário do mundo real. Ele é composto basicamente por duas minúsculas telas de TV e um conjunto de lentes especiais.

As lentes ajudam a focalizar imagens que estão a milímetros dos olhos do usuário, ajudam também a ampliar o campo visual do vídeo. Além disso, os HMDs funcionam também como um dispositivo de entrada de dados, pois contem sensores de rastreamento que medem a orientação e posição da cabeça, transmitindo esses dados para o computador. à RV objetivam estimular, eficientemente, a maior quantidade de sentidos e capturar com fidelidade os movimentos dos usuários.

do mundo real. Dessa forma, cabe ao hardware de Realidade Virtual de saída de dados estimular tais sentidos. A principal preocupação da saída dos sistemas de RV é a estereoscopia

nados na frente do usuário); a segunda categoria é composta pelos monitores de computador e sistemas de projeção. A diferença entre essas duas categorias é que, na primeira o dispositivo possui sensores para detectar os movimentos do usuário, enquanto que na segunda isso não acontece, sem contar que o rastreamento depende dos comandos do usuário via outro dispositivo de entrada.

Os vídeo-capacetes (Figura 2) são construídos, geralmente, usando três tipos de telas: os monitores de TV (CRT), os monitores de cristal liquido (LCD) - os mais usados atualmente -, e os de diodo emissores de luz orgânicos (OLED). 
Figura 2 - HMD CRT de duas telas desenvolvido na primeira geração de HMDs da NASA

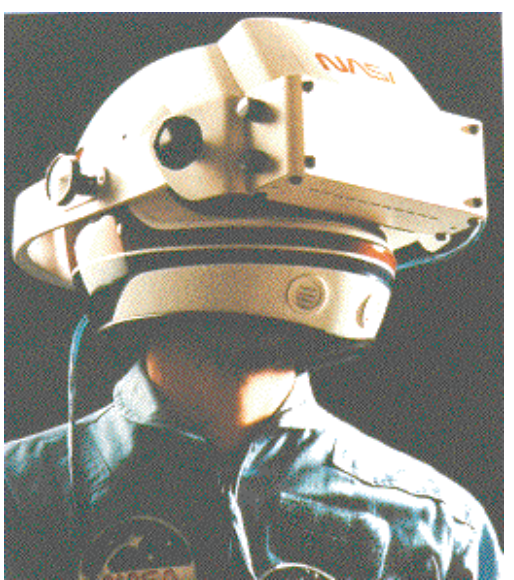

Fonte: Kalawski (1993).

\section{- HEAD-COUPLED DISPLAY (BOOM)}

Consiste de um display montado sobre um braço mecânico com um contrapeso. 0 formato do BOOM (Figura 3) permite uma fácil transição entre a visua-

Nos sistemas de RV fundamentados em monitores ou sistemas de projeção, o usuário precisa ficar constantemente olhando para a tela ou monitor além de utilizar um dispositivo de entrada de dados para que possa controlar a sua movimentação no ambiente virtual. Óculos obturadores (shutter glasses) (Figura 4) são utilizados para filtrar as duplas de imagens geradas pelo computador. 0 que isso quer dizer? Isso quer dizer que, o computador exibe, alternadamente, as imagens direita e esquerda sincronizadas com óculos que bloqueiam cada um dos olhos, o que permite ao usuário, visualizar uma imagem "saindo" da tela.

Figura 4 - Exemplo de um shutter glasses.

O som 3D tem o objetivo de proporcionar uma sensação de imersão. Da mesma forma que o ser humano possui visão estereoscópica, ele também pos-
Figura 3 - 0 sistema de display BOOM utilizado com uma estação de trabatho de alta resolução. Montado sobre um braço articulado com contrapeso

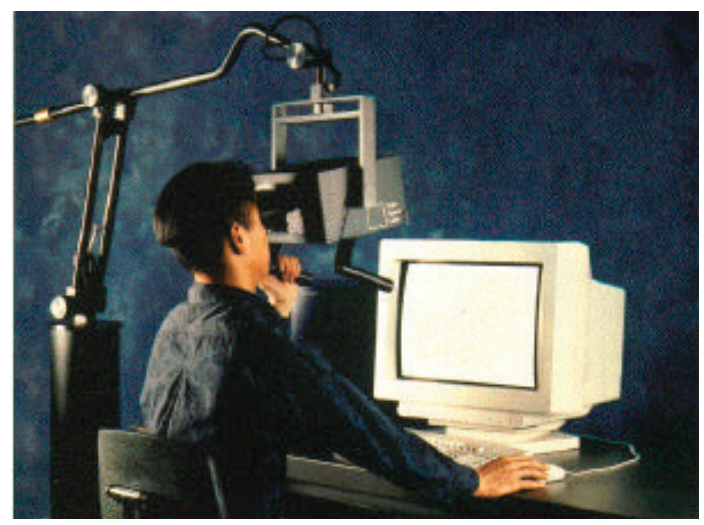

Fonte: Bolas (1994).

\section{- MONITORES E SISTEMAS DE PROJEC̣̃̃O}

lização do mundo virtual e a interação com monitores, teclados e outros dispositivos que possam estar fazendo parte do controle da simulação.

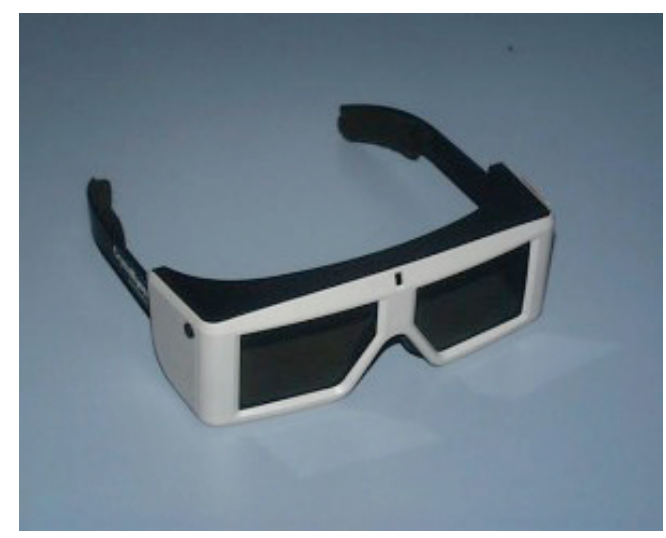

Fonte: (TV3DWITHOUTGLASSES, 2011 - on-line) 3.2 DISPOSITIVOS AUDITIVOS

sui audição estéreo que desempenha o mesmo papel, apesar de funcionar de forma diferente, do som 
3D: enganar o cérebro. Tanto que, em um sistema de som 3D perfeito, não é possível diferenciar simulação de realidade.

Existem inúmeras placas de som projetadas para ções físicas, como o tato, a temperatura e a tensão muscular. A clara diferença entre estes dispositivos e os visuais e auditivos, se encontra na necessidade de uma sofisticada interação eletromecânica com o corpo do usuário.

A utilização de dispositivos físicos em sistemas de

Reação Tátil ou Feedback Tátil é a denominação dada a sistemas que transmitem sensações que atuam sobre a pele. Esse feedback deve fornecer a sensação do toque bem como permitir ao usuário distinguir

São os sistemas que permitem as sensações de peso e pressão. Uma maneira de construir um sistema deste seria uma espécie de braço mecânico encaixada no corpo do usuário, fazendo com determinados mo-

Os dispositivos de saída possibilitam o usuário a "entrar" no mundo virtual. Mas, são os dispositivos de entrada que permitem a movimentação e a interação do participante com o mundo. Sem um dispositivo de entrada o usuário participaria experiência de RV apenas de forma passiva.

Pimentel (1995) separa esses dispositivos em duas

Os dispositivos de interação permitem ao usuário a movimentação e manipulação de objetos no mundo virtual. Exemplo: trabalhar junto com ferramentas que constroem ambientes em realidade virtual. Existem até algumas placas que permitem trabalhar simultaneamente com diversas fontes de som.

\subsection{DISPOSITIVOS FÍSICOS}

RV envolve a usabilidade de sistemas computacionais potentes e dispositivos específicos de entrada e saída. Destacam-se aqui dias classes de dispositivos físicos: reação (feedback) tátil e reação de força.

- REAÇ̃̃otÁtIL

rugosidade, temperatura, geometria e características de atrito de superfície associadas ao objeto tocado.

\section{- REACÃO DE FORCA}

vimentos the permitisse sentir o peso de um objeto no ambiente virtual.

\subsection{DISPOSITIVOS DE ENTRADA DE DADOS}

categorias: dispositivos de interação e dispositivos de trajetória.

\subsubsection{DISPOSITIVOS DE INTERACÃO}


Através da luva de dados (Figura 5) o sistema de $\mathrm{RV}$ reconhece os movimentos das mãos do usuário que a utiliza.

Figura 5 - Exemplo de uma luva de dados baseada em fibra ótica.

\section{- SENSORES DE ENTRADA BIOLÓGICOS}

Estes sensores processam atividades indiretas, como sinais elétricos musculares e comando de voz.

Em RV comandos de voz tendem a facilitar a execução de tarefas no ambiente virtual. Em contrapartida, os dispositivos que utilizam sinais elétricos musculares, detectam essas atividades por meio de eletrodos colocados sobre a pele.

\subsubsection{DISPOSITIVOS DE TRAJETÓRIA}

Este tipo de dispositivo é responsável pelo rastreamento da trajetória, conhecido também como tracking. Esses dispositivos se baseiam para operar na diferença de posição em relação a um ponto de referência. Essencialmente, existe uma fonte que emite o sinal (pode esta localizada no dispositivo de interação), um sensor que recebe este sinal, e uma caixa reguladora que processa o sinal e faz a comunicação com o computador.

\section{- APLICAÇÕES}

A todo o momento aplicações novas surgem, devido à demanda e capacidade criativa das pessoas através da RV a interação homem-maquina mudou. Devido ao avanço tecnológico de hardwares e softwares, a utilização de recursos de RV vem propiciando as empresas maior desempenho e menores custos.

A medicina tem sido uma das áreas de preferência dos desenvolvedores de RV. Nos países desenvolvidos,

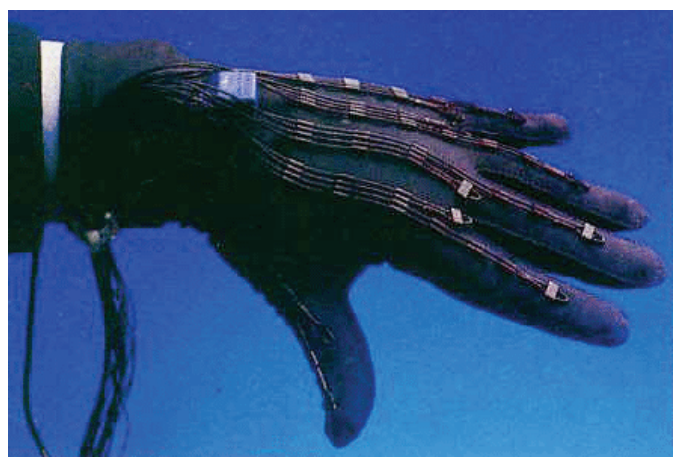

Fonte: (AICRISTI.BLOGSPOT, 2011 - on-line)

A utilização de pequenos sensores colocados sobre partes do corpo ou sobre o objeto é feita na maioria das aplicações que usam detecção de trajetória, essa técnica é conhecida como tracking ativo. 0 tracking passivo por outro lado, utiliza sensores óticos ou câmeras para "observar" o objeto e determinar sua orientação e posição.

Em oposição aos dispositivos que utilizam tracking ativo, os dispositivos de tracking passivo fazem uso de apenas um sensor para rastrear o objeto.

a RV tem sido empregada no ensino de anatomia e na simulação de operações (Figura 6). As imagens dos moldes virtuais possibilitam avaliar os órgãos tridimensionalmente, além de permitir observar a estrutura interna do órgão entre outras vantagens. (MONTERO; ZANCHET, 2003)

Devido à tridimensionalidade e a possibilidade de visualizar diferentes pontos de observação, a RV 
tem sido considerada uma ferramenta impar no que se refere à percepção, apreciação, avaliação e representação dos elementos arquitetônicos (Figura 7) em escala e à simulação de texturas e efeitos luminosos nas superfícies e volumes.

A indústria militar, há anos tem profundo interesse nessa área em decorrência de vários fatores. 0 primeiro, e mais óbvio, é a segurança de treinar um militar em ambiente simulado e controlado (Figura 8),

Figura 6: Simulação de cirurgia em realidade virtual.
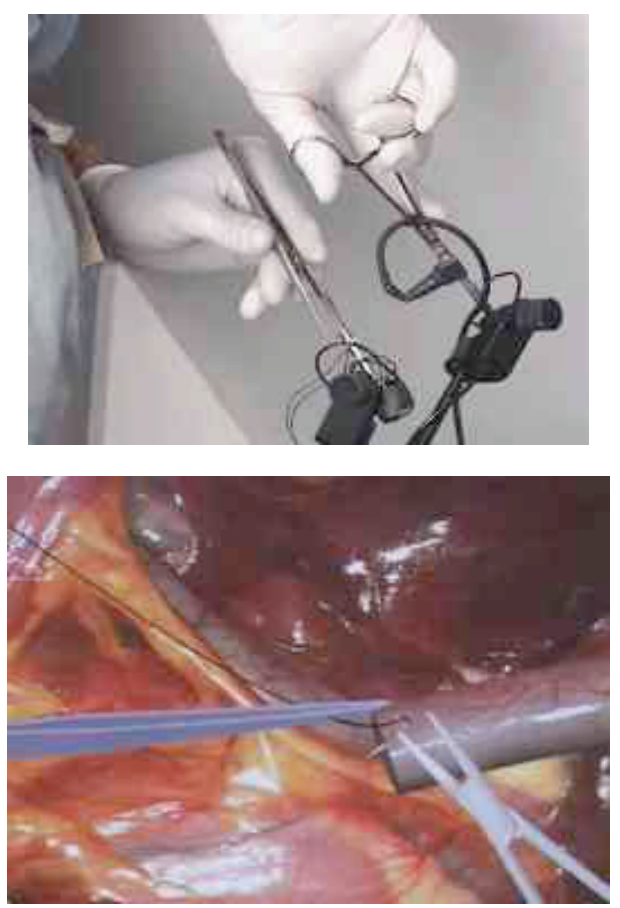

Fonte: (INFORMATICAMEDICA, 1999 - on-line) que numa situação real. Por um lado considerando a vida do piloto, por outro considerando o custo do equipamento, que geralmente chega a custar milhões de dólares. A Força Aérea, o Exército e a Marinha usam simuladores de voo para treinamento dos pilotos. As missões de treinamento podem incluir: como voar em batalha, como se restabelecer em caso de emergência ou como coordenar a sustentação no ar com operações terrestres. (INFORMATIZAÇÃO MILITAR - QC6, 2001, on-line).

Figura 7: Projeto de arquitetura, em 3D, antes da construção de uma maquete.

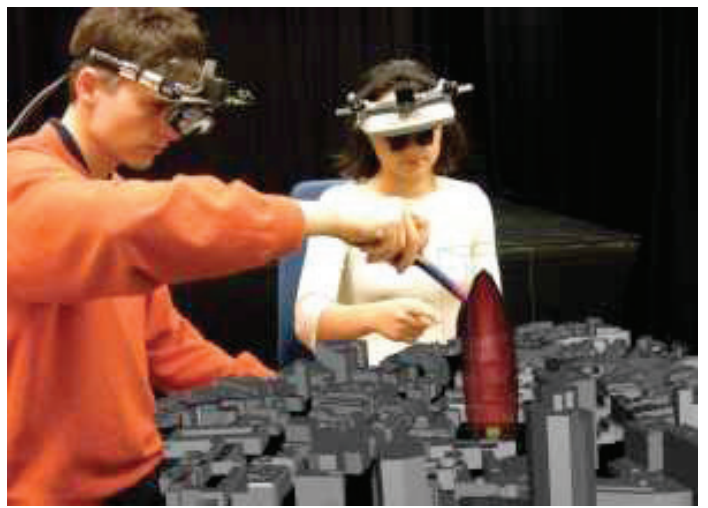

Fonte: (INOVACAOTECNOLOGIA, 2004 - on-line).

Figura 8: Militar norte americano usando HMD para treinamento de salto de paraquedas.

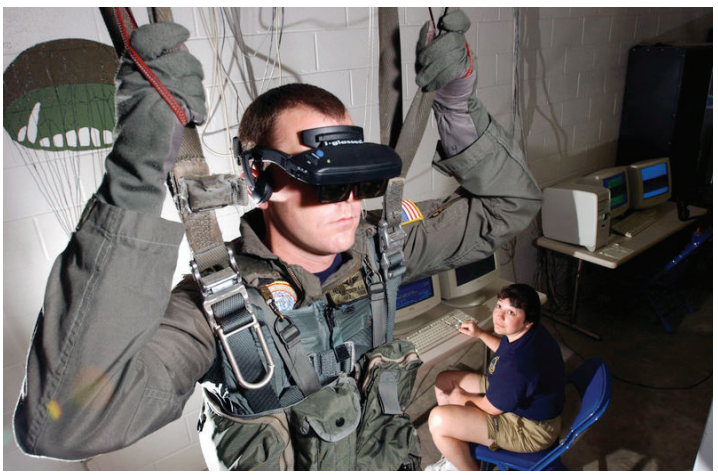

Fonte: (CSUFES20092, 2010 - on-line). 


\section{CONSIDERACÕ̃ES FINAIS}

Mediante as abordagens efetuadas no decorrer do texto, é certo afirmar que Realidade virtual é um tipo de interação com o computador, auxiliada por dispositivos como: capacetes, óculos, luvas ou trajes especiais. E apoiada na composição absolutamente realista de imagens tridimensionais, dando a ilusão de estar presente num ambiente criado pela máquina.

Observou-se ainda que as aplicações em Realidade Virtual passaram por um processo gradual de evolução. Com o aperfeiçoamento dos dispositivos de saída e entrada de dados, aliada ao grande desenvolvimento dos computadores digitais, contribuiu para a criação de aplicações potentes e voltadas para diversas áreas.

Portanto, a possibilidade de fazer um modelo virtual reproduzir exatamente as sensações geradas pelo cérebro e pelo sistema visual no contato com um objeto real, a ponto de não distinguir uma imagem do mundo real e a projeção de um mundo virtual ainda é improvável já que entre a realidade e o ambiente virtual existe uma lacuna. A dificuldade na implementação em ambientes virtuais nesse aspecto torna-se difícil devido as limitações tecnológicas, a ausência de um entendimento mais claro no que se refere a aspectos sensoriais dos ambientes virtuais e reais além da criação/utilização de novos conceitos tridimensionais que a RV possibilita.

Muito embora, os investimentos na área tenham permitido uma gradual modificação deste quadro, além disso, o avanço das pesquisas neste ramo vem proporcionando ferramentas de software e hardware cada dia mais sofisticados a um menor custo. Dessa forma, atesta-se que ainda não se pode definir um modelo completo, um protótipo que forneça toda a generalização e flexibilidade que um ambiente real exige. Com isso, atualmente, é possível apenas modelar partes da realidade com particularidades e restrições. Dessa forma, o uso de RV ainda é limitado a alguns tipos de projetos funcionais.

\section{REFERÊNCIAS}

ARAÚJO, R. B. Especificação e análise de um sistema distribuído de realidade virtual, São Paulo, Junho, Tese (Doutorado), Departamento de Engenharia de Computação e Sistemas Digitais, Escola Politécnica da Universidade de São Paulo, 1996.

BRAGA, Mariluci. Realidade Virtual e Educação, revista de biologia e ciências da terra volume 1, numero 1, 2001

BRYSON, S. Virtual reality in scientific visualization, Communications of the ACM, p. 62-71, vol. 39, n. 5, May, 1996.

CHAVES, Eduardo O. C. Virtualização da Realidade, São Paulo, Setembro/Dezembro, 1999.

COSTA, Fernando Henrique da. Simulador de bicicleta em quatro dimensões: Realidade Virtual com implementação física, Sorocaba, Dezembro, Relatório (Trabalho de Conclusão de Curso), Universidade Estadual Paulista (UNESP), 2010. 
DANTAS, Mario. Realidade Virtual. Disponível em: <http://mariodantas.files.wordpress.com/2010/02/dispositivos-de-entrada-e-saida.pdf> Acesso em: 20 nov. 2011

INFORMATIZAÇÃO MILITAR - QC6. Disponível em: <http://csufes20092.pbworks.com/w/page/5335740/ Informatiza\%C3\%A7\%C3\%A30\%20Militar\%20-\%20QC6>

INOVAÇÃO TECNOLÓGICA. Disponível em: <http://www.inovacaotecnologica.com.br/noticias/noticia. php?artigo=010150040512> Acesso em: 22 nov. 2011.

JACOBSON, L. Realidade virtual em casa. Rio de Janeiro, Berkeley, 1994.

LÉVY, Pierre. 0 que é virtual? Trad. Paulo Neves. São Paulo: Ed.34, 1996.

MACHADO, Liliane dos Santos. Conceitos Básicos da Realidade Virtual, São José dos Campos, Novembro, 1995.

MONTEIRO, Edna Frasson de Souza; ZANCHET, Dinamar José. Realidade virtual e a medicina. Scielo. Disponível em:<http://www.scielo.br/scielo.php?pid=S0102-86502003000500017\&script=sci_arttext> Acesso em: 20 nov. 2011.

PENTEADO, S. 0 mundo da quinta dimensão, Informática Exame, pp. 55-60, n. 111, junho, 1995.

PIMENTEL, K. ; Teixeira, K. Virtual reality - through the new looking glass. 2. . New York, McGraw-Hill, 1995.

PLAYNGIVE. KISTLER R. Monkey Business| Ralph Kistler. Disponível em: <http://www.playngive.com/ home/2011/8/13/monkey-business-ralph-kistler.html> Acesso em: 23 nov. 2011.

PORTO, Cristiane. Internet e comunicação científica no Brasil: quais impactos? Quais mudanças? Disponível em: <http://www.repositorio.ufba.br/ri/handle/ri/6845> Acesso em: 28 set. 2012 Salvador: Edufba. E-book.

REALIDADE VIRTUAL CONCEITOS E TENDÊNCIAS. Livro do Pré-Simpósio VII Symposium on Virtual Reality São Paulo, 19 out. 2004.

SABBATINI. Renato M. E. Informática Médica. Disponível em:<http://www.informaticamedica.org.br/informaticamedica/n0202/sabbatini.htm> Acesso em: 24 nov. 2011.

SUBTOURS. SIEBER Jan M.; KISTLER R. Monkey Business. Disponível em: <http://www.subtours.com/cms/ node/76> Acesso em: 16 nov. 2011

VON SCHWEBER, L. ; VON SCHWEBER, E. Cover story: realidade virtual, PC Magazine Brasil, p. 50-73, v. 5, n. 6, junho, 1995. 

Graduanda em Sistemas de Informação pela Universidade Salvador - UNIFACS. E-mail: gessicapr@gmail.com

Doutora Multidisciplinar em Cultura e Sociedade - Ufba. Mestre em Letras - Ufba. Professora Plena do Programa de Pós-graduação em Educação da Universidade Tiradentes - Unit. Vice-Líder e pesquisadora do Grupo de Pesquisa em Educação, Tecnologias da Informação e Cibercultura Avaliado em: XX de abril de 2013

- Unit/CNPq e pesquisadora do Grupo Comunicação, Educação e Sociedade Aceito em: XX de abril de 2013 